\title{
ESTANDARIZACIÓN DEL PROCESO DE FUNDICIÓN PARA ARENAS CON RESINAS AUTOFRAGUANTES EN LA INDUSTRIA MILITAR FÁBRICA SANTA BÁRBARA
}

\author{
(Standardization of the process of smelting for sands with self-forgeling resins in the Military \\ Industry Santa Bárbara Factory)
}

\author{
Lina Consuelo Carvajal Fernández, Laura Melisa Suárez Díaz
}

Escuela de Ingeniería Industrial, UPTC, lina.carvajal@uptc.edu.co, lauramlisa.suarez@uptc.edu.co.

(Recibido julio 13 de 2014 y aceptado septiembre 1 de 2014)

\begin{abstract}
Resumen:
El presente artículo describe la estandarización del proceso de fundición para arenas con resinas autofraguantes en la Industria Militar Fábrica Santa Bárbara. Se estudió el proceso de moldeo en la planta de fundición durante seis meses, para establecer los estándares de mano de obra y materia prima, de tal manera que permitieran evaluar de forma verídica los costos por kilogramo de pieza fundida. Se trabajaron 41 piezas del sector civil y militar a lo largo del desarrollo del proyecto. Finalmente se evaluó cada estándar tanto el de mano de obra como el de materia prima para diferentes piezas, y con los resultados obtenidos se evidenció una mejora del proceso, esencialmente en mano de obra, mientras que en materia prima los estándares no presentaron una variación significativa, en este caso se corroboró que los existentes son aplicables al proceso.
\end{abstract}

Palabras clave: resinas autofraguantes, estándar, proceso de moldeo, fundición.

\section{INTRODUCCIÓN}

La fundición es uno de los procesos más utilizados en la industria. Esta se encarga de llevar los metales hasta el punto de fusión, para que el metal adopte la forma deseada a través de un molde. Este proceso es realizado manual y mecánicamente, requiere un tiempo de realización elevado para algunas de sus etapas, como la de secado del molde y terminado de la pieza. En la actualidad, se utilizan diferentes alternativas de moldeo, uno de

\begin{abstract}
:
This article describes the standardization of the smelting process for sands with self-forgeling resins in the Military Industry Santa Barbara Factory. The molding process was studied in the Smelter for six months, to set standards for workforce and raw materials, so that would allow truthfully evaluate the costs per kilogram of casting. 41 pieces of civil and military sectors throughout the development of the project were worked. Finally both standards were evaluated, labor as raw material for different parts, with results was evidenced improved process, essentially in workforce, while in raw material standards did not show significantly change, in this case is corroborated that existing ones are applicable to the process.
\end{abstract}

Keywords: self-forgeling resins, standard, molding process, casting.

ellos es arenas con resinas autofraguantes, que permite la disminución en el tiempo de fabricación de los moldes y mejoras en el acabado superficial de las piezas; además de ser reutilizable dentro del ciclo de producción.

Para que un proceso cualquiera en la industria sea fiable y cuente con información real y a tiempo y se conozcan las cantidades de materia prima y mano de obra directa e indirecta necesarias para poder asegurar la disponibilidad y eficiencia de los medios de producción, es necesa- 
rio estandarizar. La estandarización consiste en registrar y utilizar metódicamente los mejores pasos para un óptimo desarrollo de los procesos y actividades, que genere cambio y mejora.

La Industria Militar Fábrica Santa Bárbara adquirió todo un sistema para realizar el moldeo para arenas con resinas autofraguantes, con el fin de aumentar la productividad y reducir los costos de fabricación. Con el desarrollo del proyecto, la Fábrica contará con el estudio que le servirá como pilar dentro de su sistema de gestión y le permitirá a la empresa cumplir la entrega de productos en el plazo establecido, con calidad y bajo las especificaciones técnicas establecidas.

\section{CONTENIDO}

\subsection{Metodología}

La estandarización del proceso de fundición para arenas con resinas autofraguantes en la Industria Militar Fábrica Santa Bárbara, se considera como una investigación de tipo descriptivo, puesto que no se tienen antecedentes de estudios de medición del tiempo y de mejora de métodos, y de tipo exploratorio, ya que se sometió a análisis y medición. Para realizar mejoras significativas de manera consistente dentro de una organización, es importante tener un modelo estandarizado de mejora. DMAIC es el proceso de mejora que utiliza la metodología Seis Sigma $\mathrm{y}$ es un modelo que sigue un formato estructurado y disciplinado. DMAIC consta de cinco fases conectadas de manera lógica entre sí (definir, medir, analizar, mejorar, controlar). Cada una de estas fases utiliza diferentes herramientas que son usadas para dar respuesta a ciertas preguntas específicas que dirigen el proceso de mejora.

\subsection{Descripción del proceso de fundición}

La Fábrica desarrolla sus actividades específicas administrativas y operativas, siempre bajo el concepto de calidad; para lograr esto cuenta con diferentes talleres que le permiten integrar y perfeccionar sus labores, y estos están distribuidos a lo largo de su infraestructura; algunos de estos son: fundición, mecanizados, tratamientos superficiales, calidad, mantenimiento, entre otros.

En la planta de fundición se elaboran piezas para los sectores de defensa, agrícola, construcción, minero, cementero y energético (termoeléctricas y petroleras). Esta tiene capacidad para fundir piezas entre $1 \mathrm{~kg}$. y $4500 \mathrm{~kg}$. brutos, cuenta con un horno de inducción de tres cubas, que tiene la capacidad de fundir con dos cubas de 1000 $\mathrm{kg}$ y una de $250 \mathrm{~kg}$ brutos de material. También tiene un horno eléctrico de arco con una capacidad de $2500 \mathrm{~kg}$ brutos.

a) Fabricación del modelo. Se realiza en el taller de modelos, inicia con la revisión de la orden de fabricación, se analizan los consumos de materia prima y mano de obra. Luego se hace una evaluación del plano y de la matriz operativa, donde se establecen dimensiones, sobre materiales y contracción, dependiendo del tipo de material. Se hace trazado, corte, ensamble y construcción del modelo, fabricación de caja de machos, si se requiere, acabado y pintura al modelo. Por último, una inspección y control dimensional.

b) Fabricación del molde con arenas para resinas autofraguantes. La fabricación de los moldes inicia con la activación del sistema neumático que transporta la arena nueva desde la tolva de recepción y la arena recuperada desde la tolva del shake out, hasta la tolva de los mezcladores. Se dosifica la arena en los moldes y se compacta. Después del tiempo de curado se extraen los bloques de arena de los moldes.

c) Fabricación de machos. Los machos se elaboran con la misma arena del molde y son los encargados de crear las cavidades y formas internas que necesite la pieza final. Se fabrican con la ayuda de moldes metálicos o de madera, en los cuales se vierte arena mezclada, se apisona manualmente y se deja curar la arena para formar el macho. Algunos machos requieren una capa de pintura refractaria antes de ser ubicados en los moldes.

d) Pintar, secar y sellar molde. Los bloques de arena y cajas se limpian con aire a presión para retirar impurezas. Algunas cajas requieren una capa de pintura refractaria en la base y la tapa, dependiendo del acabado superficial de la pieza. Todas las cajas llevan una capa de pintura en el sistema de alimentación para evitar desprendimiento de la arena cuando se vierta el material fundido. Se utilizan dos tipos de pintura refractaria: a base zirconio y sílice, la cual tiene como disolvente alcohol isopropílico y se utiliza para aceros al carbono; a base magnesita $98 \%$, que tiene como disolvente metanol y se utiliza para aceros al manganeso y hierros. 
e) Fusión y colada. En esta etapa, el metal se calienta por encima de la temperatura de fusión, por medio de un horno de inducción. Se utiliza chatarra cold rolled, chatarra reutilizable y diferentes ferroaleaciones para obtener la composición química y balance de cargas requeridos de acuerdo con lo establecido por el cliente. La planta de fundición también cuenta con un horno eléctrico de arco. El material es llevado desde el horno de inducción hasta los moldes por medio de cucharas, que son precalentadas para disminuir el choque térmico cuando se vierta el material. En cada uno se vierte material hasta que las mazarotas estén llenas. Se agrega una cucharada de exotérmico (ferrux) en las mazarotas para conservar la temperatura interna durante más tiempo.

f) Desmoldar pieza fundida. Después del colado, se debe esperar a que la pieza se solidifique y se enfríe en el molde, lo que puede requerir horas o días, dependiendo del tamaño de la pieza. Cuando la pieza ha solidificado, se retira la arena que contuvo el molde. El moldeo de arena con resinas autofraguantes permite que esta arena pueda ser recuperada y reutilizada en gran proporción. Las cajas se llevan a la criba del shake out, y por medio de vibración se desprende la arena que contiene la pieza.

g) Granallar pieza fundida. La pieza extraída del molde aún contiene arena en su superficie; se lleva a la granalladora y por medio de esferas metálicas (granallas), se desprende la arena. La duración de este proceso depende de la cantidad de piezas que entren al granallado en función del tamaño y geometría de la pieza.

h) Corte, alimentación, limpieza y terminado. Se inicia en la sección de corte donde se retiran los sistemas de alimentación, usando oxígeno y acetileno, o disco de corte cuando son hierros. En la zona de limpieza se pule la superficie de la pieza para dar acabado; algunas piezas presentan imperfecciones como rechupes y poros, en estos casos se debe recuperar con soldadura. Algunas piezas también requieren mecanizados para rectificar acabados superficiales y dimensiones, otras requieren masilla y una capa de pintura anticorrosiva. Finalmente, las piezas son inspeccionadas por el personal de calidad y transportadas al almacén de producto terminado.

i) Tratamiento térmico. Adicionalmente, las piezas necesitan tratamiento térmico, para mejorar sus propiedades físico-mecánicas (dureza, resistencia a la tracción, reducción de área, elasticidad); las piezas son sometidas a tratamientos térmicos como: alivio de tensiones, aus- tenización, normalizado, recocido, revenido, temple en aceite, aire y agua.

\subsection{Realización del estudio de tiempos}

El estudio de tiempos con cronómetro es la técnica más común para establecer los estándares de tiempo de un proceso. Con esta técnica de medición se toman observaciones aleatorias de los trabajadores para determinar la proporción de tiempo que gastan realizando las tareas. El estándar de tiempo es un elemento importante de información que está sujeto a tres condiciones: un operario calificado y previamente capacitado, que trabaja a velocidad y ritmo normal y hace una tarea específica. Los pasos para la elaboración del estudio de tiempos son los siguientes: dividir la operación en elementos, efectuar el estudio de tiempos propiamente dicho, determinar los ciclos por cronometrar, aplique tolerancias y tiempo estándar. (Meyer, 2007).

En la Tabla 1 se muestra la cantidad de piezas por cada etapa y en cada rango, además la clasificación especial que se les hizo por el tipo de características que presentan, para que el estándar que se calculó fuera lo más preciso posible.

Tabla 1. Cantidad de piezas por etapa del proceso, rango de peso y características de clasificación

\begin{tabular}{|c|c|c|c|c|c|}
\hline \multicolumn{6}{|c|}{ PIEZAS POR ETAPA, RANGO DE PESO Y CARACTERISTICAS } \\
\hline ETAPA & CARACTERISTICA & $\begin{array}{c}1-10 \\
\mathrm{~kg}\end{array}$ & $\begin{array}{c}10-50 \\
\mathrm{~kg}\end{array}$ & $\begin{array}{c}50-300 \\
\mathrm{~kg}\end{array}$ & $\begin{array}{c}300- \\
700 \mathrm{~kg}\end{array}$ \\
\hline \multirow{6}{*}{$\begin{array}{l}\text { FABRICACION } \\
\text { DEL MOLDE }\end{array}$} & CAJA METALICA CON & 5 & 4 & 6 & 2 \\
\hline & CAJA DE MADERA & 0 & 2 & 4 & 0 \\
\hline & CAJA METALICA CON & 14 & 1 & 0 & 0 \\
\hline & CAJA DE MADERA & 1 & 1 & 0 & \\
\hline & CON PLACA MODELO & 1 & ${ }^{1}$ & 0 & \\
\hline & CAJA MODELO & 1 & 4 & 0 & $\underline{0}$ \\
\hline \multirow{2}{*}{$\begin{array}{c}\text { PINTAR,SECAR } \\
\text { Y SELLAR } \\
\end{array}$} & CAJA METALICA & 19 & 5 & 6 & 2 \\
\hline & BLOQUE DE ARENA & 2 & 8 & 4 & 0 \\
\hline \multirow{2}{*}{ DESMOLDEO } & CAJA METALICA & 19 & 5 & 6 & 2 \\
\hline & BLOQUE DE ARENA & 2 & 8 & 4 & 0 \\
\hline \multirow{3}{*}{ GRANALLADO } & NINGUNA & & & & \\
\hline & CLASIFICACION & 21 & 13 & 10 & 2 \\
\hline & PARTICULAR & & & & \\
\hline \multirow{2}{*}{$\begin{array}{c}\text { CORTE DE } \\
\text { ALIMENTADOR }\end{array}$} & ACEROS & 19 & 12 & 9 & 1 \\
\hline & HIERROS & 2 & 1 & 1 & 1 \\
\hline \multirow{3}{*}{$\begin{array}{l}\text { LIMPIEZA Y } \\
\text { TERMINADO }\end{array}$} & GEOMETRIA I & 15 & 8 & 4 & 1 \\
\hline & GEOMETRIA II & 6 & 4 & 3 & 0 \\
\hline & GEOMETRIA III & 0 & 1 & 3 & 1 \\
\hline \multirow{3}{*}{$\begin{array}{l}\text { FABRICACION } \\
\text { DEL MACHO }\end{array}$} & MACHO PEQUEÑO & \multicolumn{4}{|c|}{13} \\
\hline & MACHO MEDIANO & \multicolumn{4}{|c|}{20} \\
\hline & MACHO GRANDE & \multicolumn{4}{|c|}{3} \\
\hline
\end{tabular}




\subsection{Controlar y registrar la cantidad de materia prima} e insumos necesarios para la fabricación de una pieza

Esta actividad se efectuó a la par con el estudio de tiempos; se controló la cantidad de materia prima e insumos que requería la fabricación de cada pieza. Durante el estudio se recopilaron los consumos para cada una de las piezas que fueron objeto de análisis, y se obtuvo la mayor cantidad de información posible. Para materias primas e insumos específicos, el proceso en el cual intervienen dificulta el cálculo de consumo por pieza o unidad. Se describirán las formas de control y registro para cada una de las materias primas y cada uno de los insumos.

En la Tabla 2 se muestran las materias primas e insumos controlados durante el desarrollo del proyecto, además los estándares que se obtuvieron luego de tabular la información.

Tabla 2. Estándares de materia prima e insumos obtenidos en el desarrollo del proyecto.

\begin{tabular}{|c|c|l|r|}
\hline \multicolumn{4}{|c|}{ ESTANDARES DE MATERIA PRIMA E INSUMOS } \\
\hline Ítem & Artículo & \multicolumn{1}{|c|}{ Descripción } & $\begin{array}{c}\text { Estandar } \\
\text { propuesto }\end{array}$ \\
\hline 1 & P330-9235 & Arena seca 40-70 nueva & 2,53110 \\
\hline 2 & & Arena seca 40-70 recuperada & 6,37350 \\
\hline 3 & P101-4096 & Resina tipo 1 autofraguante & 0,06130 \\
\hline 4 & P101-4097 & Resina tipo 2 autofraguante & 0,04770 \\
\hline 5 & P101-4098 & Catalizador para resinas 1 y 2 & 0,00760 \\
\hline 6 & P330-5211 & Pintura stripcote dr 2077 & 0,00021 \\
\hline 7 & P302-0010 & Gas propano cilindro p & 0,00010 \\
\hline 8 & P330-8896 & Metanol pureza 95\%min & 0,00420 \\
\hline 9 & P330-5473 & Pintura refrac,a base & 0,00380 \\
\hline 10 & P325-0010 & Alcohol isopropilico & 0,00410 \\
\hline 11 & P330-5473 & Pintura refrac.a base & 0,00320 \\
\hline 12 & P330-0304 & Ferrux & 0,00500 \\
\hline 13 & P330-9492 & Desescoriante materia & 0,01200 \\
\hline 14 & P316-0045 & Masa de apisonar almix & 0,00089 \\
\hline 15 & P330-0698 & Tableta refractaria u- & 0,00070 \\
\hline 16 & P330-0716 & Termocuplas desechable & 0,00520 \\
\hline 17 & P330-0315 & Granalla & 0,00679 \\
\hline 18 & P330-0029 & Acetileno & 0,00016 \\
\hline 19 & P201-0229 & Gas argon & 0,00000 \\
\hline 20 & P330-9215 & Oxigeno gaseoso & 0,00479 \\
\hline 21 & P316-0019 & DISCOS ZA24R 7"- 180x6 & 0,01172 \\
\hline 22 & P316-3697 & Disco 9" x 1/8" x 7/8" & 0,00357 \\
\hline 23 & P330-9815 & Chatarra de cobles rel & 0,06533 \\
\hline 24 & P320-4893 & Punta montada a3 & 0,00480 \\
\hline 25 & P330-0135 & Soldadura aws e-6013 $\varnothing$ & 0,00010 \\
\hline 26 & P330-0142 & Soldadura aws e-7018 $\varnothing$ & 0,00331 \\
\hline 27 & P330-0165 & Sold.fe. Aws a5.15 e s & 0,00584 \\
\hline 28 & P330-0149 & Anticorrosivo 310 pint & 0,00085 \\
\hline 29 & P330-0037 & Thiner disolvente & 0,00255 \\
\hline & & & \\
\hline
\end{tabular}

\subsection{Resultados y análisis}

2.5.1 Estándar mano de obra y análisis del estudio de tiempos. Una vez tabulada la información en los formatos de registro, se calcularon los tiempos estándar, complementando con la evaluación de los operarios y las tolerancias, para cada etapa del proceso y por pieza. Se tabularon los tiempos de operación requeridos por etapa para la fabricación de cada una de ellas; se calculó la media y la desviación estándar, con el fin de conocer el coeficiente de variación de los datos. Si el coeficiente de variación es menor o igual al $20 \%$, se dice que el promedio es representativo y que los datos son homogéneos, por tanto acepta una media aritmética; pero, si el coeficiente de variación es mayor a $20 \%$, el promedio no es representativo de los datos y estos no son homogéneos.

En el estudio, la mayoría de los datos obtuvo un coeficiente de variación por encima del $20 \%$. A estos se les aplicó una holgura por encima de la media muestral, utilizando el análisis estadístico para niveles de confianza, para que los tiempos de fabricación de los rangos se cumplan para los límites de los intervalos de peso, con el fin de asegurar que los estándares calculados funcionen adecuadamente, y que, si se presenta el caso, al calcular el tiempo de fabricación para una pieza, este no esté por debajo del real obtenido.

Una vez calculados los estándares de tiempo, se hizo una comparación entre los costos de mano de obra para fabricar un martillo para molino mediano; se compararon los tres tipos de moldeo existentes en la Fábrica y se obtuvo una reducción del 49,95\% de moldeo autofraguante a moldeo manual y una reducción del 47,48 \% de moldeo autofraguante a moldeo mecánico.

Tabla 3. Comparación del costo de fabricación para las clases de moldeo martillo para molino mediano

\begin{tabular}{|l|c|c|}
\hline \multicolumn{3}{|c|}{ REDUCCIÓN EN COSTOS POR KG } \\
\hline & MOLDEO MANUAL & MOLDEO MECANICO \\
\hline MOLDEO MECANICO & $4,74 \%$ & $0,00 \%$ \\
\hline MOLDEO AUTOFRAGUANTE & $49,95 \%$ & $47,46 \%$ \\
\hline
\end{tabular}

De la misma forma se realizó una comparación de costos de mano de obra para moldeo autofraguante con las diferentes características que se presentan en el proceso; se obtuvo una reducción del 47,75\% en los costos cuando se moldea con caja modelo; siendo la mejor opción para fabricar una pieza. 
Tabla 4. Comparación del costo por tipo de moldeo autofraguante nariz bomba bel

\begin{tabular}{|l|cc|r|}
\hline \multicolumn{4}{|c|}{ REDUCCIÓN DE LOS COSTOS/KG - MANO DE OBRA } \\
\hline \multicolumn{1}{|c|}{ TIPO DE MOLDEO } & \multicolumn{2}{c|}{ COSTO / KG } & REDUCCIÓN \\
\hline CAJA METALICA CON MODELO SUELTO & $\$$ & $2.125,15$ & $0,00 \%$ \\
\hline CAJA DE MADERA CON MODELO SUELTO & $\$$ & $1.126,42$ & $47,00 \%$ \\
\hline CAJA METALICA CON PLACA MODELO & $\$$ & $1.262,53$ & $40,59 \%$ \\
\hline CAJA DE MADERA CON PLACA MODELO & $\$$ & $1.122,97$ & $47,16 \%$ \\
\hline CAJA MODELO & $\$$ & $1.110,34$ & $47,75 \%$ \\
\hline
\end{tabular}

Es importante aclarar que los estándares finales fueron evaluados solo para las piezas objeto de estudio, esto quiere decir que la confiabilidad de la información depende de la cantidad de datos recolectados para cada uno de los rangos. De esta manera, para obtener una mayor confianza en la validación de los estándares, es necesario continuar con la recolección de información que permita que a medida que aumente la cantidad de piezas estudiadas, disminuya la variación en los datos.

2.5.2 Estándar y análisis de la materia prima e insumos. Las materias primas e insumos se calcularon con base en lo registrado durante el desarrollo del proyecto en la planta de fundición. Se tabuló la información para determinar los estándares para cada una de las piezas contraladas; se resalta que la variación por kilogramo de materia prima no es considerable. El objetivo principal del análisis se basó en validar cada uno de los estándares actualmente utilizados para la elaboración de presupuestos. Posteriormente se evidenció que no existía una forma de cálculo adecuada para su respectiva verificación. Con el estándar propuesto de materia prima se garantiza que los datos son confiables; si en algún momento la Fábrica desea evaluar nuevamente los estándares, contará con toda la información necesaria y formas de cálculo para lograrlo.

En la Tabla 5 se observa una comparación de los costos de materias primas e insumos por moldeo verde y moldeo autofraguante. La variación del costo no es significativa en casi ninguna de las líneas de fabricación; la mayor disminución la presenta la línea de fundición hierro Ni-hard y la que más aumenta es la línea de fundición hierro nodular.
Tabla 5. Comparación de los costos de materia prima e insumos para moldeo en verde y moldeo autofraguante por línea de fabricación.

\begin{tabular}{|c|c|c|c|c|}
\hline \multicolumn{2}{|c|}{ (2)3 } & \multicolumn{3}{|c|}{$\begin{array}{l}\text { PRESUPUESTO DE MOLDEO PARA UN KG DE } \\
\text { MATERIAL }\end{array}$} \\
\hline ÍTEM & DESCRIPCION & $\begin{array}{l}\text { PRESUSPUESTO } \\
\text { POR MOLDEO EN } \\
\text { VERDE ACTUAL }\end{array}$ & $\begin{array}{l}\text { PRESUPUESTO } \\
\text { MOLDEO } \\
\text { AUTOFRAGUANTE } \\
\text { PROPUESTO }\end{array}$ & DIFERENCIA \\
\hline 1 & $\begin{array}{l}\text { ACERO AL MANGANESO } \\
\text { GRADO C }\end{array}$ & 3.766 & 3.722 & $-1,2 \%$ \\
\hline 2 & $\begin{array}{l}\text { ACERO AL MANGANESO } \\
\text { GRADO A }\end{array}$ & 3.734 & 3.687 & $-1,3 \%$ \\
\hline 3 & $\begin{array}{l}\text { ACERO AL MANGANESO } \\
\text { IM + Mo }\end{array}$ & 5.705 & 5.950 & $4,3 \%$ \\
\hline 4 & ACERO 1020-1050 & 2.942 & 3.064 & $4,1 \%$ \\
\hline 5 & ACERO 4340 & 4.142 & 4.299 & $3,8 \%$ \\
\hline 6 & ACERO 4140 & 3.308 & 3.464 & $4,7 \%$ \\
\hline 7 & ACERO INOXIDABLE 304 & 8.533 & 8.213 & $-3,8 \%$ \\
\hline 8 & FUNDICIÓN HIERRO GRIS & 2.542 & 2.771 & $9,0 \%$ \\
\hline 9 & $\begin{array}{l}\text { FUNDICION HIERRO } \\
\text { NIHARD }\end{array}$ & 7.502 & 7.396 & $-1,4 \%$ \\
\hline 10 & $\begin{array}{l}\text { FUNDICIÓN HIERRO } \\
\text { NODULAR }\end{array}$ & 2.870 & 3.285 & $14,4 \%$ \\
\hline 11 & $\begin{array}{l}\text { ACERO AL MANGANESO } \\
\text { IM }\end{array}$ & 4.133 & 4.399 & $6,4 \%$ \\
\hline
\end{tabular}

\subsection{Documentación}

Para documentar el proceso de fundición para arenas con resinas autofraguantes, se diseñó una propuesta para el INOE, donde se considera el proceso de fundición en las etapas de moldeo y desmolde. Un INOE es un instructivo operacional estándar, el cual describe paso a paso las actividades desarrolladas para ejecutar una tarea, teniendo en cuenta el ciclo PHVA. Debe tener redacción sencilla con vocabulario estándar, de forma clara y detallada. Adicionalmente se incluyó lo referente al mantenimiento autónomo de la maquinaria y equipo que intervienen.

El INOE diseñado para la etapa de fabricación de moldes, considera la maquinaria, la mano de obra y las actividades necesarias para ejecutar la operación; describe los pasos que un operario debe realizar para el cumplimiento de la labor, además contempla los requerimientos de inspección y verificación del mantenimiento autónomo para el correcto estado y funcionamiento del equipo. De la misma manera, el INOE para la etapa de desmolde considera las mismas actividades y requerimientos que para la fabricación de moldes, para el shake out, elevador de cangilones, separador magnético, clasificador, refrigerador, colector de polvos y la bomba de arena recuperada. 


\section{CONCLUSIONES}

1. Se diseñó una hoja de cálculo para estándares de mano de obra especializada en el moldeo de arenas con resinas autofraguantes. De esta manera, los presupuestos que se evalúen tendrán una mayor validez, debido a que se trabajó con los datos recopilados durante el desarrollo del proyecto.

2. Al realizar la comparación de las dos referencias de piezas estudiadas en los diferentes métodos de trabajo, se evidenció que el moldeo con arenas para resinas autofraguantes permite reducir los costos de mano de mano de obra en 46,66\%, en comparación con el moldeo manual, y en $44,00 \%$, en comparación con el moldeo mecánico.

3. La planeación, organización y asignación adecuada del número de operarios en cada puesto de trabajo que permita fabricar más de una referencia de pieza a la vez en la línea de producción, evidenció un incremento del $100 \%$ en la productividad en la etapa de moldeo y, por ende, una reducción de los costos de producción.

4. Al realizar la experimentación al método de trabajo propuesto, se comprobó que el moldeo de una pieza en caja modelo, reduce el tiempo de fabricación en un 47,63 $\%$, comparado con el sistema de moldeo de esta misma en caja metálica y modelo suelto.

5. Se evidenció que los estándares de materia prima calculados para el método propuesto en cada línea de fabricación de la planta de fundición, no presentaron una disminución en el costo total por kilogramo de material fundido, aunque no ocurrió en todas las líneas; esto comprueba que los estándares establecidos actualmente son confiables.

6. Para el estándar de mano de obra de un acero al manganeso grado A, con el método propuesto, se observó una reducción en $\$ 47$, comparado con el método actual de moldeo en arena en verde por kilogramo de pieza fundida.

7. Se evidenció durante el desarrollo del proyecto que el acabado superficial y la calidad de las piezas, son mucho mejores que por los métodos tradicionales.

8. Se realizó una base de datos con las dimensiones y tipos de cajas que fueron utilizados para la elaboración de cada una de las piezas estudiadas, de esta manera la Fábrica contará con esta información para futuros presupuestos que servirán como consulta.

10. Se elaboró una propuesta para el instructivo operacional estándar INOE, el cual describe de manera ordenada y secuencial las actividades necesarias para el desarrollo de las actividades de moldeo y desmolde de arenas con resina autofraguantes, teniendo en cuenta el ciclo PHVA, además se incluye lo referente al mantenimiento autónomo de las máquinas y equipos que intervienen.

\section{RECOMENDACIONES}

Se recomienda hacer una inversión en cajas de moldeo estándar que permitan realizar los cálculos de materia prima y mano de obra adecuados y reducir los costos de producción específicamente en el proceso de moldeo y desmolde.

Se recomienda generar planes de producción de las diferentes piezas para crear líneas de producción, disminuyendo tiempos de fabricación y siendo más oportunos en las entregas de los productos al cliente.

Se recomienda evaluar y cambiar los productos al proceso de arenas con resinas autofraguantes, ya que se comprobó que el costo de materia prima no se incrementa y el de mano de obra se disminuye, permitiendo a la organización ser más competitiva.

Se recomienda implementar metodologías y técnicas para garantizar que producciones tales como sinfines, cuñas de fricción, mandíbulas, conos para trituradoras, sean fabricados por el moldeo con arenas para resinas autofraguantes.

Se recomienda evaluar la cantidad ideal de operarios en cada etapa del proceso, de manera que se pueda optimizar el recurso y disminuir los tiempos ociosos.

Se recomienda valorar un rediseño para la línea de desplazamiento del puente grúa, para que esta pueda atravesar todo el taller de lado a lado y ubicar sobre la línea dos puentes grúas; así se disminuirán los tiempos de transporte de materiales y cargas. 
Se recomienda instalar unas bandejas bajo las líneas de rodillos, que permitan la recolección de arena que desprenden los moldes durante los transportes sobre esta, de esta manera la arena que se contenga puede llevarse a la criba del shake out y retornar al sistema sin ningún inconveniente de impurezas.

Se recomienda diseñar soportes para facilitar el manejo de piezas de gran tamaño en la sección de pulido y terminado.

Se recomienda evaluar la posibilidad de automatizar la línea de rodillos, para minimizar el tiempo de los transportes y la fuerza que debe ejercer el operario sobre los moldes para el desplazamiento de estos.

Se recomienda hacer un estudio para la implementación de un tanque de oxígeno de mayor capacidad en la sección de corte y así evitar demoras en el cambio de cilindros y la continua repetitividad de esta operación.

Se recomienda efectuar la instalación de tanques de almacenamiento de mayor capacidad para las resinas y el catalizador, que permitirá disminuir los tiempos por cambio de los recipientes actualmente utilizados.

\section{REFERENCIAS}

Chaparro, S. P. (2006, agosto-diciembre). Comparación técnica de la mezcla de área para el proceso de $\mathrm{CO} 2$ y el sistema de arenas autofraguantes. Ciencia e Ingeniería Neogranadina, 16, 104112.

Meyers, F. E. (2000). Estudio de tiempos y movimientos para la manufactura ágil. (2 ed.). México: Pearson Educación.

Niebel, F. (2007). Ingeniería industrial, métodos, estándares y diseño del trabajo. México: Alfaomega.

Ocampo, J. R. \& Pavón, A. E. (2012). Integrando la metodología DMAIC de seis sigma con la simulación de eventos discretos en Flexsim. San Pedro Sula, Honduras: Universidad Tecnológica Centroamericana.

Proceso de fundición con resinas autofraguantes. http:// www.ptolomeo.unam.mx:8080/xmlui/bitstream/handle/132.248.52.100/2548/05-MPMCap2-Final.pdf?sequence $=5$

Walpole, R. E., Myers, R. H., y Myers, S.L. (1999). Probabilidad y estadística para ingenieros. (6 ed.). México: Pearson Educación. 